\title{
Criterios para mejorar la gestión y comunicación ambiental en la administración pública. Una investigación mixta en la ciudad de Madrid (España)*
}

Criteria to Improve the Environmental Management

and Communication in Public Administration. A Mixed Investigation in the City of Madrid (Spain)

Recibido: 1 de marzo de 2015 | Revisado: 1 junio de 2015 | Aceptado: 1 agosto de 2015

\author{
Mireya Palavecinos ** \\ Universidad de La Frontera, Chile \\ CONCEPCIÓN PIÑEIRO **** \\ MARÍA José DÍAZ ***** \\ Universidad Autónoma de Madrid, 28049, Madrid, España \\ JORGE ULLOA ****** \\ Universidad de La Frontera, Chile
}

doi:10.11144/Javeriana.up14-4.cmgc

Para citar este artículo: Palavecinos, M., Ulloa, J., Piñeiro, C., \& Díaz, M. J. (2015). Criterios para mejorar la gestión y comunicación ambiental en la administración pública. Una investigación mixta en la ciudad de Madrid (España). Universitas Psychologica, 14(4), 1459-1472. http://dx.doi.org/10.11144/ Javeriana.up14-4.cmgc

* Los datos presentados en el artículo, corresponden a datos parciales obtenidos a través de Tesis doctorales, realizadas por la Dra. Palavecinos (2014) y la Dra. Piñeiro (2011) en programas doctorales de la Facultad de Psicología y Ecología de la Universidad Autónoma de Madrid, España y gracias al trabajo de equipo del Convenio FGUAM y Ayuntamiento de Madrid durante los años 2006 al 2013.

***mireya.palavecinos@ufrontera.cl

*** Departamento de Ecología.

\section{RESUMEN}

Se presentan dos estudios complementarios que tienen como objetivo analizar la gestión ambiental del municipio de Madrid, desde una perspectiva que considera diversas miradas: la ciudadana, la de los técnicos y la experta. Se ha utilizado el método mixto, que considera tanto técnicas cualitativas como cuantitativas para la recolección de datos. Entre otras, se recurrió al análisis documental, revisión de quejas ciudadanas presentadas al sistema de Sugerencias y Reclamaciones (SyR) del ayuntamiento $(N=932)$ y la realización de entrevistas a expertos y gestores locales. Los resultados entregan datos que permiten verificar problemas en la gestión y comunicación municipal que perpetúan los conflictos ambientales. En las conclusiones, se valora el rol de la educación ambiental como complemento de otros mecanismos inherentes a la administración (gestión, legislación, inversión). Se generan propuestas para mejorar la gestión ambiental desde la mayor participación y la comunicación con los ciudadanos, como forma de desarrollar acciones educativas y una gestión ambiental más eficiente desde la administración pública.

Palabras clave

gestión ambiental; administración pública; participación ciudadana

\section{A B S T R ACT}

Two complementary studies, in which experts, technicians and citizens are consulted regarding communication campaigns and environmental management conducted by the City Council of Madrid. A mixed method is developed (qualitative and quantitative techniques) with interviews, educational campaigns and review of citizens' complaints to the municipality $(N=932)$. The results highlight some problems within local management and communication that maintain environmental conflicts. The conclusions seek to increase the value of environmental education and their different strategies, consistent in complementarity with other mechanisms inherent to public administration (management, legislation, investment). Some proposals are delivered to improve the management of resources and increase efficiency by the development of educational campaigns and communication with the citizens of this government. Keywords

environmental management; public administration; citizen participation 
En las últimas décadas el gobierno local de la ciudad Madrid ha aumentado los recursos materiales y técnicos destinados a la gestión ambiental del municipio. Sin embargo, estas medidas no se traducen en soluciones a los problemas ambientales existentes en la ciudad.

Desde las autoridades locales se promueven nuevas actuaciones que intentan mejorar la gestión de la sostenibilidad municipal, en coherencia con las exigencias de la normativa europea respecto de la gobernanza ambiental. El objetivo fue analizar el contexto local, desde una perspectiva múltiple: la ciudadana, la técnica y la de los expertos. Para ello, se realizó una investigación, desarrollada en el municipio, solicitada desde el Departamento de Educación para la Sosteniblidad del Ayuntamiento de Madrid, a la Universidad Autónoma de Madrid. El proceso fue llevado a cabo por un equipo interdisciplinar, conformado por personal investigador de la universidad, de los departamentos de Ecología y Psicología social.

Se presenta la importancia de la participación como un proceso de aprendizaje individual y colectivo (participación deliberativa) y la comunicación y educación ambiental, como una estrategia de gestión que busca generar procesos que fortalezcan la gobernanza, considerando la implicación ciudadana como fundamental en la mejora de los problemas ambientales que se enfrentan en la administración pública (Palavecinos et al., 2008).

El presupuesto municipal destinado a problemas ambientales se fue incrementando en los últimos años, fundamentado por informes del Gobierno de la ciudad de Madrid, donde se expresaba que entre los aspectos que empeoran en la calidad de vida de las personas en la ciudad, luego del tráfico, estaban los problemas vinculados a la calidad del ambiente urbano, tales como la limpieza y contaminación, superando incluso los problemas de seguridad ciudadana y desempleo.

Diversos estudios subrayan la necesidad de mejorar el flujo de información para consolidar una adecuada comunicación, para la toma de decisiones y la participación de la ciudadanía en la gestión de sus contextos (Tábara, 2006; Zube, 1984). Por ello, las medidas de gestión emprendidas por el Gobierno local están en algunos casos, asociadas a actuaciones de comunicación ambiental o acompañada de canales de comunicación entre la Administración y sus ciudadanos. Sin embargo, la falta del encuentro fluido entre ambos agentes es patente, si se observa la ineficiencia de las medidas de gestión de la sostenibilidad de la ciudad, especialmente en materia de gestión de residuos y limpieza diaria. Ante este contexto, se plantea este trabajo sobre el que se desarrollan las siguientes preguntas de investigación:

- ¿Qué características presenta la comunicación de la gestión ambiental de la ciudad de Madrid?, ¿Cuáles son las barreras para la solución de los conflictos ambientales locales?

- ¿Qué recomendaciones ofrecen las personas y los expertos para mejorar las intervenciones de comunicación ambiental (CA, en adelante) asociadas a la gestión ambiental municipal?

Para abordar estas preguntas, se estructura una investigación aplicada, organizada en dos estudios que se describen a continuación.

\section{Estudio 1. Análisis de las estrategias de comunicación ambiental para mejorar la implicación ciudadana en la gestión de residuos y la limpieza, en la ciudad de Madrid}

La importancia de la CA crece dentro de un área de investigación y gestión más amplia y consolidada, como es la educación ambiental (EA). El análisis de esta última disciplina y sus iniciativas tiene un mayor recorrido desde el ámbito académico, sin embargo, la CA no ha sido suficientemente estudiada, siendo sus intervenciones escasamente evaluadas en relación con la gestión. Esta trayectoria coincide en el Gobierno local de Madrid.

Se considera la CA en forma de campaña, programa o plan estratégico de comunicación de carácter público o colectivo, que emplea distintos medios y soportes (spots o anuncios audiovisuales, banners, cartelería, folletos, cuñas de radio, etc.). El principal objetivo de la CA es el cambio de factores psicológicos y/o sociales (valores, actitudes, comportamientos, opiniones, hábitos, significados, etc.) 
actuales hacia unos más proambientales (Piñeiro, 2008). Esta definición reconoce un amplio rango de iniciativas.

\section{Objetivos}

Los principales objetivos de este estudio son:

- Conocer la opinión de diversos expertos en diferentes áreas de gestión y comunicación ambiental para planificar iniciativas de CA y el modo como se incluyen criterios como la ética, la participación y la motivación.

- Concluir en recomendaciones para plantear las iniciativas de CA en el municipio de Madrid en la gestión de residuos.

Para abordarlos se concretan las siguientes preguntas de investigación:

1. ¿Cuáles son las estrategias y herramientas para diseñar, planificar y evaluar mejor las iniciativas de comunicación ambiental en gestión de residuos?

2. ¿Qué barreras deben superar las iniciativas de CA?

3. ¿Qué papel tienen la ética, la participación y la motivación en la comunicación ambiental?

\section{Metodología}

Para este estudio, se plantea un diseño metodológico cualitativo centrado en entrevistas semiestructuradas individuales, en pareja, grupales y un grupo de discusión. La muestra está formada por personas expertas $(N=30)$, de las cuales dos de ellas intervienen en dos técnicas distintas (entrevista individual y grupo de discusión), por lo que es $N=30$ y no $N=32$. Estas han sido elegidas a través de un muestreo de tipo intencional, bajo unos criterios de selección establecidos previamente (Tabla 1), que son sumativos para cada técnica.

El diseño metodológico comienza con un grupo de discusión exploratorio para determinar las principales variables que se van a analizar en las entrevistas en profundidad. De este modo, se configura un guión de entrevistas que recoge dichas variables cuya síntesis aparece en la Tabla 1.

Tras la recogida de datos, la trascripción es estrictamente literal de un lenguaje oral, como afirma Vallés (2002). El análisis de la información es sociohermenéutico; postula una interpretación del texto en contexto, basada en el estudio de las líneas argumentales o discursivas.

\section{Resultados}

Respecto a la primera pregunta de investigación, existe consenso entre las y los expertos consultados: todos recomiendan que las actuaciones de sensibilización ambiental desde una única institución, deben estar orientadas a la búsqueda de un objetivo de gestión ambiental, utilizando estrategias de educación, comunicación y participación que lo apoyen. Para el diseño de estrategias de CA, en apoyo a la gestión, se propone una serie de pasos

TABLA 1

Resumen técnicas, variables y criterios de selección en muestreo intencional

\begin{tabular}{|c|c|c|c|}
\hline Técnica & $\mathrm{N}$ & Criterios de selección sumativos & Variables analizadas \\
\hline $\begin{array}{l}\text { Grupo de } \\
\text { discusión }\end{array}$ & 7 & $\begin{array}{l}\text { Reconocida trayectoria en diferentes } \\
\text { disciplinas vinculadas con la CA: gestión } \\
\text { ambiental, psicología social y publicidad. }\end{array}$ & $\begin{array}{l}\text { Condicionantes previos al diseño de una } \\
\text { intervención de CA } \\
\text { Criterios para mejorar el diseño, evaluación y } \\
\text { planificación de la CA }\end{array}$ \\
\hline \multirow{4}{*}{$\begin{array}{l}\text { Entrevista } \\
\text { grupal (5) y } \\
\text { entrevista en } \\
\text { pareja (2) }\end{array}$} & \multirow[t]{4}{*}{7} & \multirow{4}{*}{$\begin{array}{l}\text { Diversidad en el ámbito profesional: } \\
\text { académico, administración pública, } \\
\text { empresa privada y organizaciones sin ánimo } \\
\text { de lucro. }\end{array}$} & Pasos en la preintervención \\
\hline & & & Carencias en la planificación \\
\hline & & & Debilidades internas \\
\hline & & & Errores en los mensajes \\
\hline $\begin{array}{l}\text { Entrevista } \\
\text { individual }\end{array}$ & 18 & $\begin{array}{l}\text { Expertos en ética, motivación y } \\
\text { participación ambiental. }\end{array}$ & $\begin{array}{l}\text { Relevancia de la ética, la participación y la } \\
\text { motivación en la CA }\end{array}$ \\
\hline
\end{tabular}

Fuente: elaboración propia 
que incluyen: la mejora de la planificación de las actuaciones de CA, la detección de las necesidades de CA asociadas al contexto y al segmento de la población objetivo y el desarrollo de un prediseño y un plan de evaluación desde el inicio, considerando experiencias previas de esa población, de otras iniciativas de CA o de la literatura específica ya existente.

Otros aspectos subrayados en los resultados son las denominadas "barreras a la CA". Son errores comunes en las prácticas de CA, los cuales son identificados por los entrevistados a modo de lista de chequeo que se debe evitar. Estas barreras se presentan a continuación, junto con literatura científica que confirma la existencia de dichos errores.

Los resultados contribuyen a abrir el debate sobre la necesidad de reorientar las intervenciones de CA. Hay que asumir la complejidad de los contextos de intervención y el papel que juegan otros factores externos, sobre los que se debería profundizar en futuros trabajos de esta línea de investigación como la emprendida en este estudio.
Respondiendo a la tercera pregunta de investigación, en el análisis de resultados aparecen variables vinculadas a la ética, a la motivación y a la participación que toman roles de interés en la CA. Tienen una potencialidad reconocida por los expertos relevantes en la búsqueda de la transformación social. Existe consenso, en el caso de la participación, en que una campaña sin participación provoca rechazo a largo plazo. Sin embargo, incluir la participación de forma adecuada también requiere de criterios de calidad en los procesos. Por ello, algunos expertos rechazan el uso de una demandante participación e implicación ciudadana, para todos los procesos y áreas de gestión ambiental.

Para los entrevistados, la ética también juega un importante papel en la calidad de las iniciativas de CA. Algunos abusos derivados del "marketing verde" de actuaciones comerciales en comunicación (como el green washing) necesitan ser revisados, así como también es necesario debatir sobre el planteamiento ético de la CA implementada desde las instituciones públicas,

TABLA 2

Orientaciones en diseño, evaluación y planificación de actuaciones de CA

\begin{tabular}{|c|c|}
\hline \multirow{6}{*}{$\begin{array}{l}\text { 1. Condiciones de partida } \\
\text { (6 condiciones) }\end{array}$} & $\begin{array}{l}\text { Complementariedad entre política, gestión, participación y comunicación hacia la } \\
\text { sostenibilidad: integrar la comunicación en cada proceso. }\end{array}$ \\
\hline & Carácter estratégico: abanico de instrumentos. \\
\hline & Presencia del pensamiento ecológico: arquitectura filosófica. \\
\hline & Capacidad y principio de autocrítica. \\
\hline & Interrelación desde el primer momento y en equipo interdisciplinar. \\
\hline & Contemplar las dificultades y la viabilidad. \\
\hline \multirow{11}{*}{$\begin{array}{l}\text { 2. Pasos en la pre- intervención } \\
\text { (11 pasos) }\end{array}$} & Detectar necesidades de comunicación (demanda interna y externa). \\
\hline & Diagnóstico del reto ambiental (interno y externo). \\
\hline & Diagnóstico poblacional y seguimiento permanente. \\
\hline & Rol de la comunicación respecto a otros instrumentos. \\
\hline & $\begin{array}{l}\text { Establecer metas (de medio plazo) y objetivos (a corto plazo), supuestos de cambio } \\
\text { e indicadores de evaluación asociados a los objetivos. }\end{array}$ \\
\hline & $\begin{array}{l}\text { Estudio de experiencias previas del promotor de la CA, de otros agentes y de la } \\
\text { literatura científica. }\end{array}$ \\
\hline & Prediseño de la propuesta: ideas-fuerza, diversidad de técnicas y empatía. \\
\hline & Determinar momento de inicio y duración. \\
\hline & $\begin{array}{l}\text { Capitalizar el "poso" que se ha generado después de otras intervenciones en la } \\
\text { misma dirección y articularlo. }\end{array}$ \\
\hline & Evaluación previa (atractivo y comprensión) y previsión de la evaluación. \\
\hline & Completar el diseño con planificación y recursos creativos. \\
\hline
\end{tabular}

Fuente: elaboración propia 
TABLA 3

Barreras de la comunicación ambiental

\begin{tabular}{|c|c|c|}
\hline \multirow{6}{*}{$\begin{array}{l}\text { Carencia de planeación } \\
\text { ("Ausencias") }\end{array}$} & $\begin{array}{l}\text { No tener las técnicas apropiadas de medición antes de } \\
\text { comenzar la campaña. }\end{array}$ & $\begin{array}{l}\text { Futerra y Pnuma (2005); } \\
\text { Castro (2005) }\end{array}$ \\
\hline & $\begin{array}{l}\text { Carencia de autocrítica: "la gente no participa o no } \\
\text { escucha" o "el problema es el político que no entiende } \\
\text { esto". }\end{array}$ & $\begin{array}{l}\text { Wynne (1995); Hesselink et } \\
\text { al. (2007) }\end{array}$ \\
\hline & $\begin{array}{l}\text { Carencia de planeación y claridad en la forma de } \\
\text { comunicar: problemas circunstanciales pueden generar } \\
\text { campañas contradictorias. }\end{array}$ & Futerra y Pnuma (2005) \\
\hline & Ausencia de diagnóstico y evaluación. & $\begin{array}{l}\text { Sundseth (2004); Hesselink } \\
\text { (1999) }\end{array}$ \\
\hline & Carencia de una aproximación inicial y profesionalismo. & Hesselink (1999) \\
\hline & No existencia de coherencia ambiental. & Futerra y Pnuma (2005) \\
\hline \multirow{3}{*}{ Interferencias internas } & $\begin{array}{l}\text { Objetivos no explícitos pueden desviar los objetivos de } \\
\text { la CA. }\end{array}$ & Hesselink (1999) \\
\hline & $\begin{array}{l}\text { La tiranía de la visibilidad (ser guiado por lo que es más } \\
\text { noticioso y no necesariamente lo más efectivo). }\end{array}$ & García López (2000) \\
\hline & $\begin{array}{l}\text { Carencia de coordinación entre entidades con intereses } \\
\text { comunes y mensajes contradictorios. }\end{array}$ & Futerra y Pnuma (2005) \\
\hline \multirow{9}{*}{$\begin{array}{l}\text { Errores asociados a los } \\
\text { mensajes }\end{array}$} & Mensajes generales o banales para problemas complejos. & $\begin{array}{l}\text { Calvo y Gutiérrez (2007); } \\
\text { Castro (2005) }\end{array}$ \\
\hline & $\begin{array}{l}\text { Mensaje a toda la audiencia.: " si esto es para todos, no es } \\
\text { para mí". }\end{array}$ & $\begin{array}{l}\text { Futerra y Pnuma (2005); } \\
\text { Beltrand-Org-(2004) }\end{array}$ \\
\hline & $\begin{array}{l}\text { Desde un extremo a otro: catastrofismo o mensajes } \\
\text { idílicos, conectados a un falso optimismo (para ocultar } \\
\text { problemas o dificultades). }\end{array}$ & $\begin{array}{l}\text { Futerra y Pnuma (2005); } \\
\text { Sundseth (2004) } \\
\text { Montero (2007) }\end{array}$ \\
\hline & Anuncios como propaganda institucional. & $\begin{array}{l}\text { García }(2000) \\
\text { Castro }(2005)\end{array}$ \\
\hline & $\begin{array}{l}\text { Dar una respuesta preconcebida y no dejar espacio para la } \\
\text { reflexión. Paternalismo. }\end{array}$ & $\begin{array}{l}\text { García (2000); Hesselink } \\
\text { (1999) }\end{array}$ \\
\hline & Usar datos no verificados. & Sundseth (2004) \\
\hline & $\begin{array}{l}\text { Usar lenguaje técnico (tecnicismos o endogamia } \\
\text { comunicativa). }\end{array}$ & $\begin{array}{l}\text { Futerra y Pnuma (2005); } \\
\text { Sundseth (2004) } \\
\text { Hesselink (1999) }\end{array}$ \\
\hline & $\begin{array}{l}\text { Culpar a los individuos y asignar excesiva responsabilidad } \\
\text { en los ciudadanos, que genera un efecto de } \\
\text { "desresponsabilización". }\end{array}$ & Pol, Vidal y Romeo (2001) \\
\hline & Etiquetar o reforzar estereotipos. & Fueyo (2002) \\
\hline
\end{tabular}

Fuente: elaboración propia

las organizaciones ambientalistas, las asociaciones y los movimientos sociales. Paradójicamente, la motivación es un elemento que no aparece en el discurso de la CA, siendo una parte de transformación imprescindible desde modelos de intervención socioambiental como el modelo de las cuatro esferas (que apuntan a cuatro cuestiones clave para la construcción de iniciativas de CA: racionalidad, emotividad, funcionalidad e influencia social), planteado por Pol et al. (2001).
Siguiendo este modelo, una de las premisas asumidas es que el cambio necesita la esfera de la información ligada a la racionalidad. Muchos expertos consideran que la CA, tradicionalmente, se centra en la deliberación racional como llave necesaria en la lucha frente a la crisis ambiental. Sin embargo, hay otras personas entrevistadas que consideran la esfera de la emoción como clave aprendida del sector de la publicidad. Por otro lado, hay expertos que subrayan la influencia de los grupos primarios 
(influencia y deseabilidad social). Por último, la funcionalidad implica tener en cuenta la mayor relación entre la gestión y la CA que señalan los expertos. De forma general, se indican elementos de las cuatro esferas del citado modelo, que deberían ser considerados desde las iniciativas de CA, según los objetivos de gestión y el contexto.

\section{Estudio 2. Análisis de las denuncias ciudadanas por temas ambientales}

A pesar de la alta inversión de recursos del ayuntamiento, en los últimos años, los departamentos de limpieza y residuos reciben cada día más quejas (aproximadamente 300 diarias). Las denuncias son recibidas a través del sistema de Sugerencias y Reclamaciones (SyR), implementado por la gestión institucional como parte de los mecanismos legales exigidos en la normativa española (gobernanza), para favorecer la participación ciudadana y como control de calidad para la gestión pública. Considerando este canal de comunicación entre la ciudadanía y el Gobierno local, se plantea la siguiente pregunta de investigación: iqué información le entregan estas denuncias a la gestión municipal?

Las denuncias por conflictos ambientales detectados en la ciudad, que afectan la calidad de vida de las personas, se presentan en el formato de la "Ficha de reclamación", creado por la institución para estos efectos, y es presentado en los canales institucionales para su tramitación (formato digital página web institucional, vía telefónica número 010, oficinas recepción de quejas, defensor del pueblo). Este instrumento de comunicación entre la ciudadanía y el ayuntamiento, parece ser una importante fuente de comunicación respecto de los problemas ambientales y las necesidades que la ciudadanía tiene en esta área. Por tanto, se les puede considerar un potente mecanismo para mejorar la gestión ambiental institucional.

\section{Objetivos}

- Identificar los principales conflictos ambientales asociados a la limpieza y residuos urbanos en
Madrid, denunciados por la ciudadanía ante el ayuntamiento.

- Reconocer barreras y facilitadores para el comportamiento proambiental en este contexto local.

- Proponer criterios para un nuevo estilo de gestión ambiental que incorpore las necesidades de la ciudadanía, para favorecer la sustentabilidad en la ciudad.

\section{Participantes}

Esta investigación recoge datos de dos fuentes:

a) Técnicos y personal administrativo, a cargo de las tareas en los departamentos de limpieza y recogida de residuos del ayuntamiento (2 gerentes, 6 del personal administrativo a cargo recepción de las denuncias y 2 inspectores de campo).

b) 932 Fichas registro denuncias ciudadanas, sistema SyR recibidas en los departamentos, entre los meses de enero a marzo. Las fichas fueron seleccionadas según los criterios preestablecidos para el estudio, tales como: datos de identificación y descripción de la situación completa, fecha ingreso, denuncia comprobada por inspectores y respondida desde la institución, ficha ingresada en los sistemas formalmente establecidos por la institución (sistemas SIGSA, S-400, SyR).

\section{Procedimiento}

El método se estructura a través de etapas, en las que la investigación se organiza según tres elementos definidos en el Modelo de Evaluación Ambiental propuesto por Zube (1984). El modelo sugiere centrarse en la evaluación desde el nivel institucional, desde los problemas ambientales detectados y en los niveles de participación presentes. Se recurre al método mixto (Creswell, 2013), para lo cual se inicia un análisis cualitativo de los contenidos expresados en las fichas de denuncia y en las transcripciones de las entrevistas realizadas a diferentes miembros de la organización. Durante el análisis de los resultados, las distintas respuestas a las preguntas abiertas se fueron categorizando y contrastando entre sí. Se llevó a cabo un proceso de análisis de contenido, a través 
de la elaboración de categorías en un procedimiento de ida y vuelta de información con los participantes, vía correo electrónico. Podemos definir el análisis de contenido como una técnica de investigación cuya finalidad es la descripción objetiva, sistemática y cuantitativa del contenido manifiesto de la comunicación o de cualquier otra manifestación de la conducta (Martin, 2014).

Finalmente, se realizó un análisis cuantitativo de los datos que entregan las fichas, con un análisis estadístico de los resultados, que corresponde a un enfoque exploratorio de extracción de relaciones de dependencia funcional de tipo jerárquico entre una variable respuesta y un conjunto de variables influyentes, desarrollado por Breiman, Freidman, Olshen y Stone (1984). En el caso del empleo de una variable de respuesta cualitativa, este método recibe el nombre de segmentación jerárquica. Una de las versiones más genéricas en cuanto a las escalas de medida de las variables empleadas y el equilibrio en la selección de los segmentos encontrados, corresponde a la metodología denominada: CART (Classification And Regression Trees). Esta técnica fue empleada en este estudio, para seleccionar las variables que presentaron la mayor representación de la separabilidad de los puntajes de los diferentes dominios observados en los estudiantes. En el caso del algoritmo empleado, este, al igual que en el caso de los análisis exploratorios multivariantes de interdependencia, presentó la posibilidad de imputación de datos faltantes, a partir de los registros que contaban con vectores de información completa para todas las variables (Breiman et al., 1984).

\section{Resultados}

Los resultados se presentaron considerando los objetivos del estudio. Por tanto se identificaron los conflictos ambientales asociados a la limpieza y residuos, denunciados por la ciudadanía, reconociendo las barreras y facilitadores para el comportamiento proambiental de las personas y proponiendo criterios para un nuevo estilo de gestión ambiental.

\section{Análisis organizacional: entrevistas y documentación}

Durante esta etapa, es posible obtener la perspectiva del gestor del problema: datos relacionados con el funcionamiento de la institución, canales de información, flujo de tratamiento de las denuncias ciudadanas (desde la recepción hasta la respuesta al denunciante) y la perspectiva institucional respecto del tratamiento de los conflictos ambientales en la ciudad.

\section{Facilitadores}

La organización es muy compleja y de gran tamaño. Considerando esta situación, su estructura y cultura organizacional se destaca por tener una clara delimitación de funciones, en los departamentos parte del estudio se cuenta con recursos humanos calificados, sus recursos económicos son considerados suficientes para las tareas asignadas y se tiene procedimientos legales claros (normativa) que define muy bien los parámetros con los que se debe establecer la comunicación con la ciudadanía que presenta quejas o denuncias por conflictos ambientales (sistema de tratamiento de las denuncias); todos factores que actúan como facilitadores para el cumplimiento de sus funciones.

\section{Barreras}

Por otro lado, algunas barreras fueron observadas: persisten sistemas muy complejos en el tratamiento de los datos, con escasas oportunidades de coordinación entre los departamentos y entre los miembros al interior de cada uno de ellos. Las etapas involucradas en el tratamiento de las denuncias se realizan de forma aislada, sin retroalimentación entre ellas. Los resultados no llegan a quienes realizan las tareas o quienes toman decisiones en la gestión ambiental. Los contenidos de denuncias o quejas son analizados en forma asilada en cada estamento, transformados solo en datos estadísticos, sin ser priorizados ni analizados, sino como número de incidencias recibidas. Por tanto, existe información valiosa para la gestión que no se comu- 
nica, que se pierde y así se perjudica la resolución eficiente de los conflictos ambientales detectados por la ciudadanía y se desaprovecha también la opción de valiosos insumos para la elaboración de campañas de comunicación y educación ambiental dirigida a las personas, como forma de generar un círculo virtuoso.

\section{Análisis de las denuncias ciudadanas}

En este estudio, se analiza la perspectiva ciudadana de los conflictos ambientales en la ciudad. El diagnóstico hecho por la revisión de las denuncias en las fichas institucionales permite clasificarlas en tres tipos: las que "requiere información" a la administración, representado por el $1.3 \%$ de los casos. Las que dan "sugerencias a la gestión" con el $8.4 \%$ y las "denuncias" propiamente tal y con el mayor porcentaje, un $90.3 \%$ de las incidencias analizadas. La mayoría de ellas son presentadas por personas independientes, $79.8 \%$, las restantes por organizaciones ciudadanas. Si consideramos el género, el $44.4 \%$ son mujeres y el $55.6 \%$ hombres denunciantes. $\mathrm{Al}$ tener en cuenta el territorio donde ocurren, las denuncias se distribuyen de forma heterogénea en los 21 distritos de la ciudad, centrándose en algunos de la periferia y el centro de la ciudad. Cerca del $21 \%$ de las personas indican que deben hacer más de una denuncia, ya que, en una primera instancia, no reciben soluciones a sus problemas.

\section{Conflictos ambientales denunciados}

Al clasificar los diferentes tipos de problemas, se encuentran 14 categorías o conflictos ambientales, los que, según su frecuencia en las denuncias, se distribuyen como sigue: 1) "limpieza de calles" con $32.1 \%$. 2) "acumulación de residuos" con un $15.6 \%$. 3) "mantenimiento de parques y plazas" con $14.4 \%$ y 4 ) estado de las "fachadas" (rayados, grafitis, cartelería y publicidad) representado por el $10 \%$ de los casos. Los resultados son presentados en la Figura 1.

\section{Factores psicosociales vinculados a las denuncias}

Se identifican diversos motivos por los que las personas presentan sus denuncias. En ellas se indican necesidades y preocupaciones, principalmente por las consecuencias o implicancias psicosociales atribuidas a la existencia de estos conflictos ambientales. Se considera que deterioran la calidad de vida en la ciudad e impiden satisfacer necesidades que la ciudadanía está manifestando como expectativas. Por sus características y estructura, las denuncias parecen articularse como un tipo particular de comportamiento proambiental, escasamente estudiado a la fecha, involucrando la necesidad de participación social (Palavecinos et. al, 2008; Palavecinos, 2014 ).

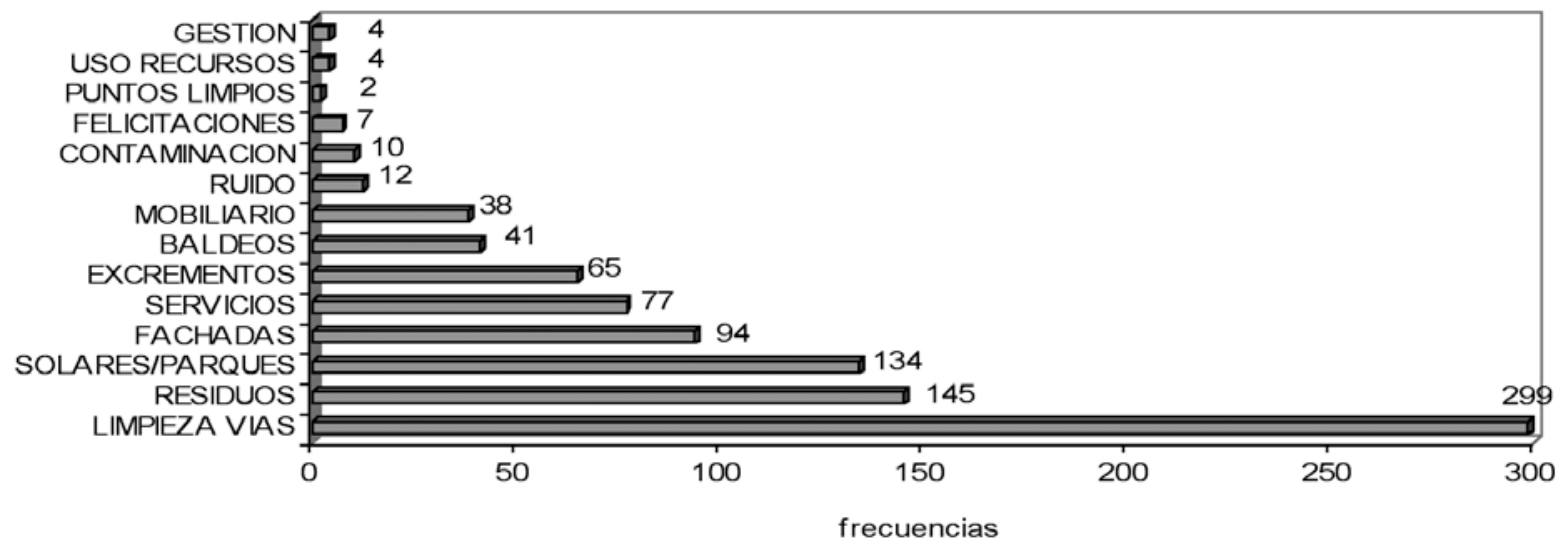

Figura 1. Temas de conflictos denunciados por frecuencia $(n=932)$.

Fuente: elaboración propia 


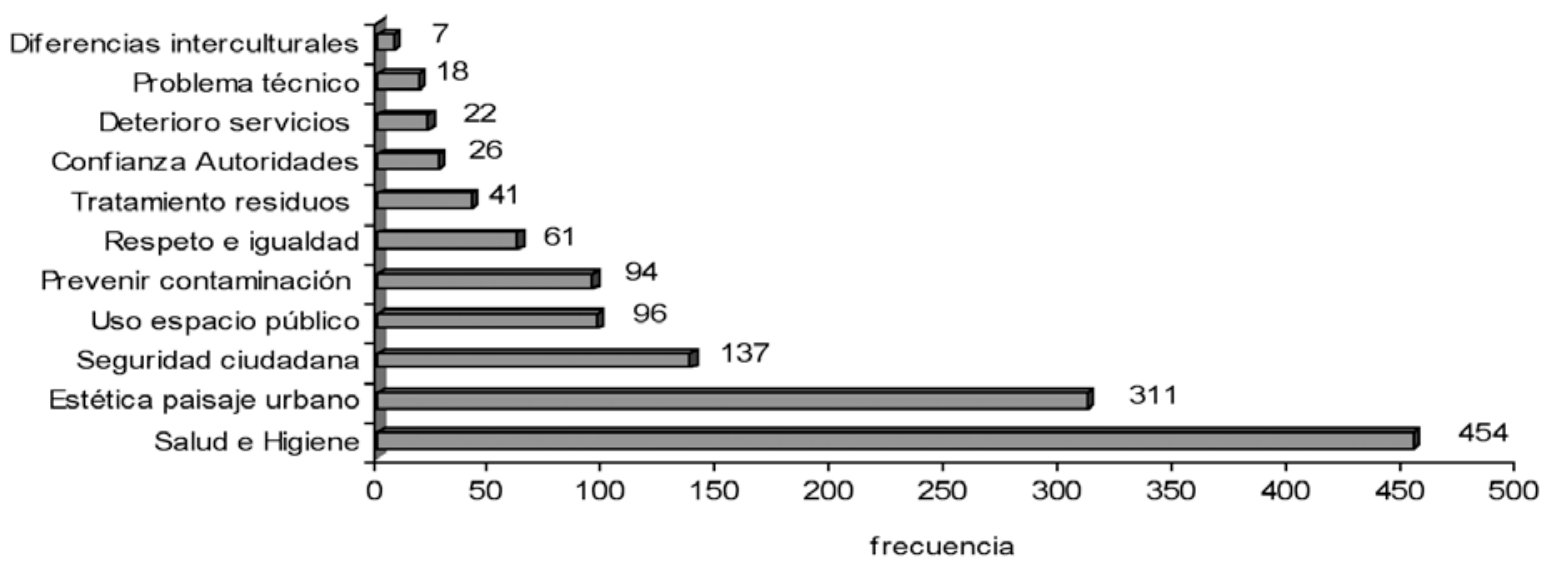

Figura2. Razones ciudadanas para la presentación de denuncias en frecuencias $(n=932)$.

Fuente: elaboración propia

\section{MODELO COMUNICACIÓN AYUNTAMIENTO - CIUDADANÍA}

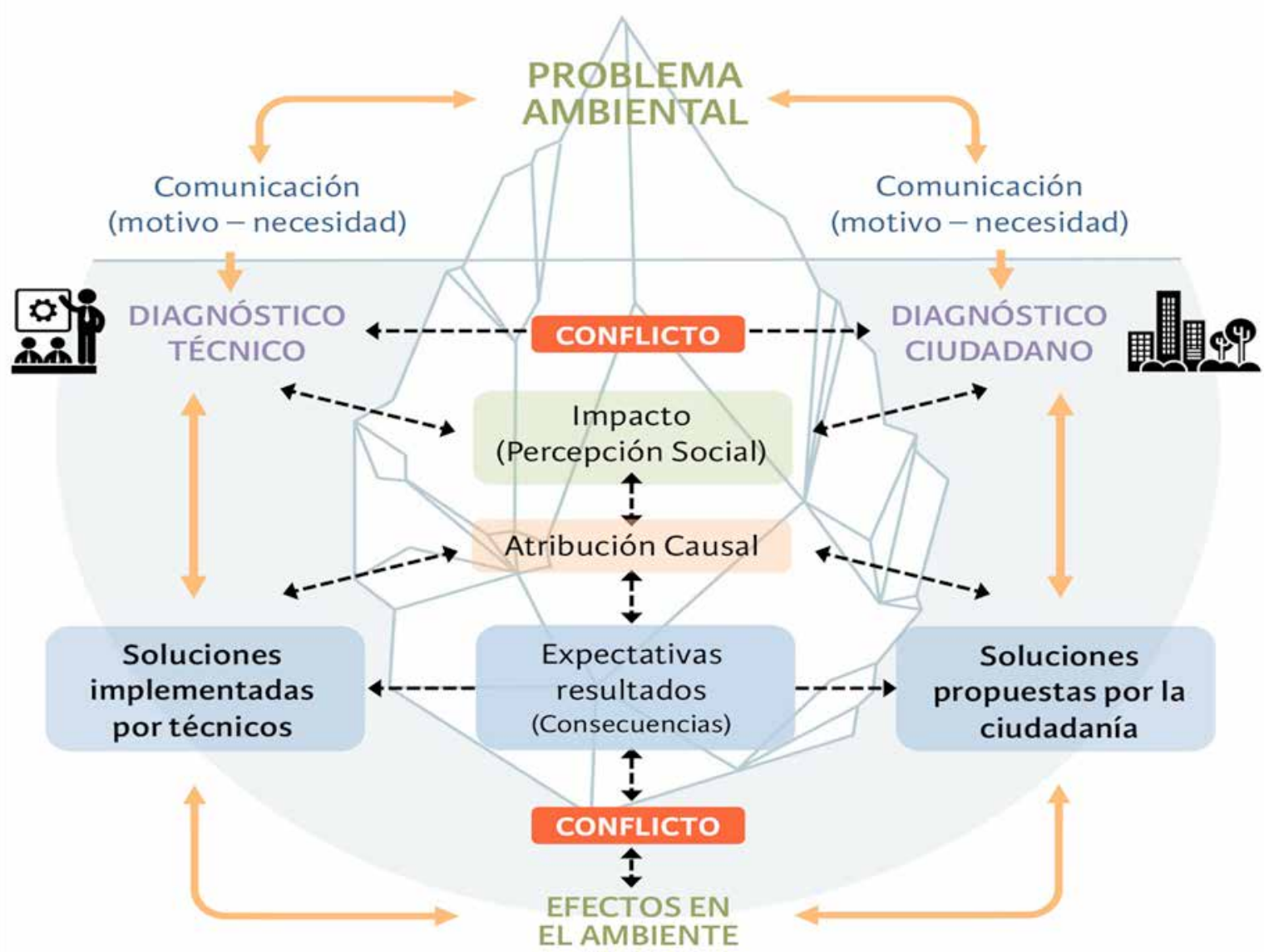

Figura 3. Modelo gestión y comunicación.

Fuente: Palavecinos (2014) 
Las denuncias expresan preocupaciones a raíz de los conflictos ambientales en la ciudad; se clasifican según frecuencia, como sigue: "consecuencias para la salud pública" (48.7\%); "necesidad de estética en espacios urbanos" (33.7\%); "inseguridad ciudadana" (14.6 \%) como efectos de la polución en la vida de las personas; la necesidad de poder hacer "uso de los espacios públicos" (10.3\%) para la vida en sociedad; necesidad de "prevenir" (9\%) consecuencias o daños para la comunidad; se exige "respeto e igualdad" (6.5\%) para los ciudadanos, ya que se considera que la contaminación afecta principalmente a los barrios y sectores más pobres en la ciudad. Por último, surgen algunas categorías con representación menor, pero que reflejan preocupación y exigencia por el "tratamiento de los residuos", especialmente los más tóxicos y complejos; la necesidad de que exista "confianza en las autoridades", "contar con herramientas técnicas" adecuadas y el que en la gestión se consideren elementos vinculados a las "diferencias interculturales". Esto puede verse en la Figura 2.

\section{Propuestas a la gestión}

Considerando estos resultados, una gestión ambiental eficiente según la ciudadanía, debería ser desarrollada en un marco institucional caracterizado por la coherencia y confianza en las autoridades que la implementan. Una gestión debe considerar las expectativas y necesidades ciudadanas en su diseño y no solo la visión de los expertos y técnicos. Se debiera desarrollar un acercamiento preventivo, considerando las características tanto de las personas como de los territorios a los que va dirigida, para el diagnóstico de los problemas ambientales que debe resolver. En suma, una gestión ambiental moderna debe actuar bajo estándares de responsabilidad social y ética de la administración pública, evidenciando respeto por los derechos de todos y asegurando igualdad de calidad de servicios de la ciudadanía a la que sirve. En la gestión, se deben asignar roles tanto a los ciudadanos como a los técnicos y las autoridades responsables del mejoramiento de la calidad de vida en las ciudades y cuidado del medio.
A modo de resumen, se presentan algunas de las conclusiones más relevantes de este estudio, en la siguiente figura (Figura 3).

\section{Conclusiones}

La ciudad de Madrid ha llegado a concentrar la mayoría de los problemas ambientales que usualmente se han diagnosticado en las grandes urbes modernas (problemas de movilidad, de diferentes tipos de contaminación, espacios públicos saturados, gran gasto energético para poder funcionar, entre otros). Problemas que, por su magnitud y características, son muy complejos de analizar y de solucionar, y afectan diariamente a las personas que allí viven.

Esta situación ha provocado que sus habitantes se sientan desconformes de vivir en un entorno con esas características. El malestar se ha ido acumulando y canalizando hacia diferentes vías, en especial hacia el ayuntamiento y sus autoridades, instancia responsable de la gestión ambiental de la ciudad. En particular, la molestia se ha expresado con la presentación de gran cantidad de reclamaciones (quejas) hacia los departamentos vinculados a la gestión ambiental, como son; Limpieza, Calidad y Residuos. Este malestar en general no es tenido en cuenta en las campañas de CA.

Considerando los resultados ya expuestos en ambos estudios, se describen las principales conclusiones para la investigación. La metodología utilizada en esta investigación, muestra el valor de las investigaciones aplicadas. $\mathrm{Al}$ recurrir a datos que las mismas instituciones poseen, pero que no logran ser analizados para que retroalimenten a la gestión institucional. Esto asume un valor aún mayor, si se trata de la administración pública, como es el caso, por el tipo de temáticas que gestiona y sus las implicancias directa en la vida de las personas.

Una gestión ambiental eficiente debería ser desarrollada en un marco institucional caracterizado por la coherencia, en un ambiente de confianza con las autoridades que implementan la gestión, que considere la participación ciudadana en su diseño y desarrolle un acercamiento preventivo, consis- 
tente con la responsabilidad social y la ética en la administración pública, por tanto, que evidencie el respecto por el medio ambiente y el rol de los ciudadanos y autoridades en su cuidado.

Las barreras de comunicación entre los ciudadanos y el ayuntamiento generan un conflicto de gestión, que ayuda a mantener los problemas y la preocupación. Estos se deben superar para evitar la presentación de un círculo vicioso de mayor deterioro del medio ambiente.

Si la comunicación es unilateral (información), ni la persuasión ni el diálogo serán posibles, porque las demás opiniones o la retroalimentación de los ciudadanos no será considerada. Por tanto, la CA debe ser diseñada teniendo en cuenta la opinión ciudadana, que es recogida a través de diferentes mecanismos, como los sistemas de sugerencias y reclamaciones.

El estado afectivo que tiene la persona producto de la insatisfacción, al percibir problemas de gestión, es consecuencia del proceso de evaluación o comparación entre la actuación institucional y las expectativas personales del ciudadano/a. Por la importancia que la situación tiene para los denunciantes, o bien por las expectativas no cumplidas, el estado de ánimo que se genera en las personas por el problema ambiental es de molestia y/o preocupación. Son estos procesos los que están entonces en el origen o motivación de la decisión de denunciar o de presentar una queja. Esta hipótesis requiere ser profundizada en estudios posteriores. Es posible considerar el comportamiento de queja ambiental, no solo como una conducta que ayuda a la protección del ambiente, sino también como una importante posibilidad de monitoreo desde la perspectiva del bienestar social de las personas. Este permite mejorar las condiciones de vida y desigualdad en la población que las implementa, elemento que no siempre ha sido suficientemente destacado en los estudios o intervenciones, pero de gran relevancia social.

Para involucrar a las y los ciudadanos, debemos asegurarnos de que todos los interesados sean integrados y representados en el proceso, estimulando el nivel de responsabilidad dada a los participantes. Esto es posible con campañas de CA que impli- quen la apertura de un proceso de participación ciudadana.

En atención a los resultados, aparece como una necesidad vital la incorporación de procesos de capacitación a los funcionarios públicos en temas como: educación ambiental, desarrollo sustentable, rol ciudadano en las tareas proambientales, aportes del control de calidad del servicio y participación social.

En un contexto aplicado, los ayuntamientos pueden jugar un rol vital en el involucramiento de sus ciudadanos para alcanzar el nivel de desarrollo sustentable adecuado. Se pueden desarrollar propuestas para una gestión ambiental participativa y líneas de intervención basadas en la interrelación entre comunicación y participación ambiental. Surge la necesidad de destacar el rol promotor que pueden jugar las instituciones públicas en esta tarea.

La generación de políticas ambientales eficientes, necesita fundamentarse en estudios y diagnósticos reales, in situ. Políticas diseñadas con estos criterios, son más eficientes en la resolución de los problemas ambientales. Estas políticas solo pueden ser aplicadas mediante programas de intervención dirigidos por instituciones responsables y considerando la implicación de la gente en el problema, desde la fase de diseño hasta la evaluación del mismo (Zube, 1984).

Los procesos de participación deben ser diseñados sin sobresaturar al público. Es importante distinguir entre información, comunicación y participación tanto en la práctica de las instituciones como en la de los gobiernos locales. En información unos hablan y otros escuchan, mientras que en la comunicación un flujo de información ocurre en todas direcciones y afecta a todas las partes involucradas. En el proceso de participación, herramientas de la comunicación producen propósitos y programas de acción que distribuyen conocimientos, objetivos, significados y responsabilidades específicas entre los interesados.

De este modo, la comunicación para el cambio social anima a una mejor relación entre los componentes del sistema, promueve acuerdos sociales y requiere de participación pública en los 
procesos de información. Tábara (2006) defiende dos dimensiones en la participación pública: a) un grado de involucramiento de diferentes agentes sociales (nivel de demanda de atención, esfuerzo y participación) y b) un grado de empoderamiento y responsabilidad, el nivel en el cual los diferentes actores sociales son capaces de entrar a los procesos de evaluación y decisión.

Nuestras conclusiones indican la necesidad de reconsiderar el valor de la opinión y el respeto por los derechos de las personas que viven los conflictos ambientales, para así desarrollar procesos de gestión, educación, comunicación y participación ambiental, pertinentes y más eficientes. Sus diversas estrategias deben buscar establecer un funcionamiento coherente y complementarlo con otros mecanismos, tales como, controles de calidad, normativa, legislación e inversión adecuadas.

\section{Referencias}

Breiman, L., Freidman, J., Olshen, R., \& Stone, C. (1984). Classification and regression trees. Monterey, CA: Wadsworth \& Brooks.

Castro, R. (2005). Más que palabras. Comunicación ambiental para una sociedad sostenible. Valladolid: GEA.

Creswell, J. W. (2013). Research design: Qualitative, quantitative and mixed methods approaches (4. ${ }^{\mathrm{e}} \mathrm{ed}$.). Londres: SAGE.

Fueyo, A. (2002). De exóticos paraísos y miserias diversas. Publicidady (re)construcción del imaginario colectivo sobre el Sur. Barcelona: Icaria.

Futerra Comunicaciones de Sustentabilidad \& Programa de las Naciones Unidas para el Medio Ambiente. (2005). Comunicando la sustentabilidad. Cómo producir campañas públicas efectivas (Guía de comunicación DTI/0731/PA). http://www.futerra. org/downloads/Guide_Spanish.pdf

García López, M. (2000). "Publicidad y medio ambiente: cuestiones para el debate". Comunicación audiovisual y medio ambiente. De la Maya, R. et al. Málaga, Junta de Andalucía. Consejería de Medio Ambiente. pp:29-38. I.S.B.N.84-607-0645-1. Editorial: Junta de Andalucía, Sevilla.
Hesselink, F. (1999). La comunicación sobre conservación de la naturaleza. 10 errores frecuentes. En F. Heras \& M. González (Coords.), 30 Reflexiones sobre educación ambiental. Madrid: Ministerio de Medio Ambiente.

Hesselink, F., Goldstain, W., Van Kempen, P., Garnett, T. \& Dala, J., (2007). La Comunicación, Educación y Conciencia Pública (CEPA). Una caja de herramientas para personas que coordinan las Estrategias Nacionales de Biodiversidad y los Planes de Acción, UICN, Montreal.

Martín, R. (2000). Estilos de vida y ahorro energético: una visión psicosocial del papel de la difusión de innovaciones tecnológicas (Tesis doctoral inédita). Facultad de Psicología, Universidad Autónoma de Madrid.

Martín, R. (2014). Análisis de contenido. Madrid: Universidad Complutense de Madrid. Recuperado: https:// www.uclm.es/profesorado/raulmmartin/Estadistica_Comunicacion/AN\%C3\%81LISIS\%20DE\%20 CONTENIDO.pdf

Palavecinos, S. M. (2014). El significado del comportamiento de queja en temas ambientales ante la administración municipal: el caso de Madrid (Tesis doctoral inédita). Psicología social y Organizacional, Universidad Autónoma de Madrid.

Palavecinos, S. M., Martín, R., Díaz, M. J., Piñeiro, C., Benayas, J., Alonso, L. E., \& Jiménez, A. (2008). Comportamiento proambiental: estudio del comportamiento de queja ciudadana. Implementación de un sistema participativo de gestión ambiental en Madrid. Revista de Psicología Social, 23(2), 243-257.

Pol, E., Vidal, T., \& Romeo, M. (2001). Supuestos de cambio de actitud y conducta usado en las campañas de publicidad y los programas de promoción ambiental. El modelo de las 4 esferas. Estudios de Psicología, 22(19), 111-126.

Sundseth, K. (2004). LIFE-Nature: Communicating with stakeholders and the general public. Best practice examples for Natura 2000. Luxemburg: European Comission.

Tábara, D. (2006). Los paradigmas culturalista, cualitativo y participativo en las nuevas líneas de investigación integrada del medio ambiente y la sostenibilidad. En R. Castro \& M. Jiménez (Coords.), Persona, sociedad y medio ambiente: perspectivas de la 
investigación social de la sostenibilidad (pp. 84-104). Sevilla: Egondi Artes Gráficas S. A.

Vallés, M. (2002). Entrevistas cualitativas. Cuadernos Metodológicos, 32. Madrid: Centro de Investigaciones Sociológicas.

Wynne, B. (1995). Public understanding of science. En S. Jasanoff, G. E. Markel, J. C. Petersen \& T. Pinch
(Eds.), Handbook of science and technology studies (pp. 361-388). Londres: SAGE.

Zube, E. H. (1984). Environmental evaluation: Perception and public policy. Belmont, CA: Wadsworth Publishing Company. 
